\title{
Rope Climbing Robot with Surveillance Capability
}

\author{
Kanza Zafar \\ Department of Computer Engineering, Sir Syed University of Engineering and Technology, Karachi, Pakistan \\ E-mail: kanza.zafar@gmail.com \\ Ibrahim M. Hussain \\ Department of Computer Engineering, Sir Syed University of Engineering and Technology, Karachi, Pakistan \\ E-mail: ibrahimh@ssuet.edu.pk
}

\begin{abstract}
In the past different engineers and researcher developed robots capable of climbing for various purposes. In this paper we have developed a robot capable of rope climbing in both horizontal and vertical direction. Furthermore, the robot has the ability to perform surveillance using a camera mounted on top of the robot. The quality of the transmitted video from the camera to the computer is clear and stable. Hence the developed robot is a good choice for surveillance purposes. In addition, it can be used to traverse floors of a building. It uses an IR sensor to sense strips attached at each floor. Once the strips are sensed, a dropping mechanism is activated in which a specific object is dropped to the targeted floor or location. The robot can work in automatic mode or manual through RF signals from an RF transmitter. Finally the robot is cost effective compared to many other developed robots for rope climbing.
\end{abstract}

Index Terms - Robot, Rope Climbing, Surveillance, Microcontroller, Drop Box

\section{Introduction}

Modern robotics are moving towards manifold changes and with their integration with computing powers; such robotic elements become effective tools to solve many real time issues which humans fail to do [1]. The image of robots varies widely with researchers, engineers, and robot manufacturers. However, it is widely accepted that today's robots used in industries originated in the invention of a programmed material handling device.

The robotic domain has many endless applications in various fields including medical, engineering, inspection, automation and military. Domain based robots have various different tasks and objectives to perform. For instance military based robots have to perform critical tasks on or off the battle field. Bomb diffusion responsibility is one such example [2]. On the other hand medical robots are used for surgical maneuvers and operation based tasks. Tele-robots used for surgery are prime example of such robots [3]. Such robots require accuracy and precision in performing the required tasks. Similarly there are many domain based robots capable of performing generalized or specialized tasks that ranges from simple to very complex tasks. Surveillance and rescue are two complex tasks which can be performed by various robots and on different domains. These two tasks can be seen in military, engineering, security and many other robots.

Surveillance is an area of great interest which has been a targeted research domain by many researchers and engineers. Many algorithms, techniques, systems, deployments and infrastructures have been developed and proposed in the past $[4,5]$. Emerging fields such as image processing, computer vision, arterial intelligence and many more play an important role in tackling problems associated with security and surveillance [6, 7].

Most surveillance robots have vision capabilities to monitor the surrounding in hunt for the desired object or just of general monitoring of a specific area. For this reason when we talk about surveillance robots, one essential component which is associated with such robots is a camera which acts as a main tool for keeping track of the targeted object under surveillance for detection and recognition. Camera positioning, stability, quality and distance coverage are very important parameters which need to be considered before mounting a camera on a surveillance robot as indicated by authors in [8].

Similar camera requirements are necessary for rescue based robots. Search and rescue operation with the help of a camera mounted on a mobilized robot is again another active research area explored by various researchers and engineers [9]. Positioning and structure of the robot play an important role in rescue based operations. Robots have to be designed in such a manner to tackle the upfront situations and conditions in which they are deployed in. Usually rescue operations take place with difficult environmental and terrain conditions. Obscurity, altitude, surface and other 
challenging difficulties are common issues in rescue operations. Robots should be capable of handling such difficulties and for these robots camera positioning and quality play an important role for achieving the desired goal.

It is worth mentioning here that advance surveillance or rescue robots incorporate some amount of intelligence and decision making capabilities such as object detection and recognition. The objective of such robots is not merely in video and life stream transmission of the surrounding but rather to analyze and visualize a scene and make decision making in the form of object detection, classification, scene analysis and object tracking [10].

With all the features a particular rescue or surveillance robot can have, one major and mandatory requirement this category of robot should exhibit is a stable camera position with stable and clear vision. Stability of camera (i.e. vision) depends on the position of the camera mounted on a robot and the movement of the robot itself. For instance a static robot with no movement has a stable scene view of the surrounding. A moving robot has less stability compared to a static robot. The stability and quality of the scene is very much coupled with the amount and way of movement of the robot. It is well known that robots can have different movements but mainly these movements can be classified into five major categories: vertical/horizontal (i.e. line), rotation, curve, vibration and free movement. Free movement being the most complex as there is no specific direction of movement. On the other hand, line movement is the simplest of the lot but still camera stability is important and need to be controlled effectively during robot movement. Many researchers worked on line robots and proposed techniques for surveillance along with stability of the camera [11].

In this paper we design and implement a simple and cost effective robot which has a vertical and horizontal movement with stable vision and can be used for surveillance and rescue based applications. Specifically this robot has the capability of rope climbing vertically and horizontally. In addition the robot is capable of transmitted live feed through a wireless transmitter to the server for further processing or decision making. Note that this paper does not discuss processing aspects of the scene being transmitted by the robot.

Rest of the paper is organized as follows: in section 2 various rope climbing robots are discussed along with our proposed system for rope climbing robot. Hardware and software descriptions are presented in Section 3 with the interconnection and functionality of individual components. Result and performance analysis is given in section 4. Finally conclusion and future work is given in section 5 .

\section{Design of the Proposed Rope Climbing Robot}

In the past engineers developed various robots capable of rope climbing which can be used for various purposes. In [12] rope climbing tasks were achieved by implementing a gait and method for traversal of a rope in the horizontal and vertical cases. Different number of modules and different methods of gripping the rope in each case were proposed. In another paper researchers developed an auto-climbing robot for cleaning glass walls with a complicated curved surface and targeting specifically the National Grand Theatre of China. The robot autonomously can climb and clean the outer walls shaped in a half-ellipsoid [13]. Some times when a robot is designed, some conditions are not taken into account and functionally of such robots become inefficient in those conditions. Researchers in [14] proposed a method based on online static equilibrium analysis to control the movement of a multi-legged rope climbing robot especially in complex conditions.

In all rope climbing robots two aspects are important, grip on the rope and smoothness while movement. These two characteristics are important to carry a weight with a robot and easily to maintain an overall stability of the robot. Our designed robot is planned to climb ropes vertically and horizontally and can be manually controlled through a wireless transceiver regardless of the nature of the rope or characteristics such as size of the rope, whether the rope vertical or horizontal directional and whether the rope is having some obstacles like knots or bend at some points on the rope. The robot is planned to have, the design is simple as possible and overall cost is minimized for use the robot in practical situations.

The robot can be used for rescue operations, military operations, scientific researches operate on danger areas for humans and specific rope climbing operations can be achieved by the proposed robot. It can also provide ease to constructors; enable them to monitor the whole environment at stationary position. This Rope Climbing Robot with Surveillance Capability basically provides luxury to the builders of high under-construction buildings and provides live streaming (videos) of its surrounding on every floor to the output devices. It is best suitable for those areas whenever the task is risky, dull, dangerous, dirty and difficult to accomplish by a human beings efficiently. This Robot, on the other hand will always keep them well aware of the whole environment.

The key features of our proposed robot can be summarized as follows: rope climbing, surveillance capability using a camera, weight carry, weight drop, pausing mechanism between floors, controlling via remote control and automatic controlling. Furthermore, key components used in constructing the robot are microcontroller, motors, camera, sensors, RF transmitter, relays and power supply.

Various robot snaps are shown in Fig. 1 while the overall system design and working is shown in Fig. 2 
and works as follows: the mechanical robot itself is fixed on the rope through grippers and contains various robot controlling hardware components including a wireless transceiver for transmitting video streams from a camera mounted on the robot and for receiving signals from the user in case of manual control. Hence this robot works in two modes, manual and automatic. In automatic mode movement is pre-calibrated and calculated in advance. For instance in automatic mode of operation, programming of the microcontroller is done in such a manner that a constant pre-defined timer is set for the robot to pause. This is effective way for an automatic monitoring of floors. If the distance to be traveled by the robot between floors in known, then it is possible to program the robot in such a manner to automatically pause at each floor for specific time duration. This is useful in automatic floor surveillance or inspection. If the robot is working in auto mode, then only one way signal in the form of video feed of the area or floor to be monitored is transmitted to the server station. In addition there is an object drop box capable of dropping an item if needed. An item could be a tool for workers working on a particular floor or a mask for people trapped inside building floors caught on fire. There are various useful applications of the drop box associated with our proposed robot. Again this drop box can be controlled and programmed in such a way so that automatic object drop takes place.

In contrast to the automatic approach, manual approach gives flexibility to the user to manually control the robot and the drop down box. This is useful when the robot climes a dynamic structured building or place. Also note that our propose robot does not only move vertically but also horizontally. There are two wheels which are on to $\mathrm{p}$ of the rope to be climbed which moves in parallel with the robe. There is no chance of misbalance of the robot structure while movement. Also note that in horizontal movement, only a single rope is used and both wheels are positioned in such a manner that the overall robot structure is in hanging position. This mechanism is useful when considering dropping an object in a deep area between to high structures as shown in Fig. 3. In our design the robot can move up to any height but the diameter of the rope is fixed. Its structure is designed in such a way that in can carry load of approx $1 / 2-1 \mathrm{~kg}$ and drop that load at our desired location. In addition the circuitry is made in such a way to provide quickest response with maximum efficiency.

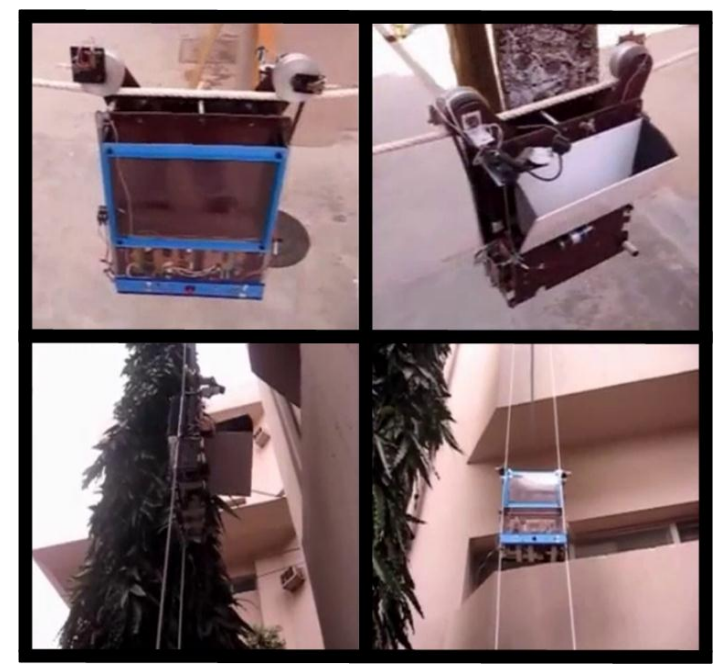

Fig. 1: The designed robot under vertical and horizontal movements

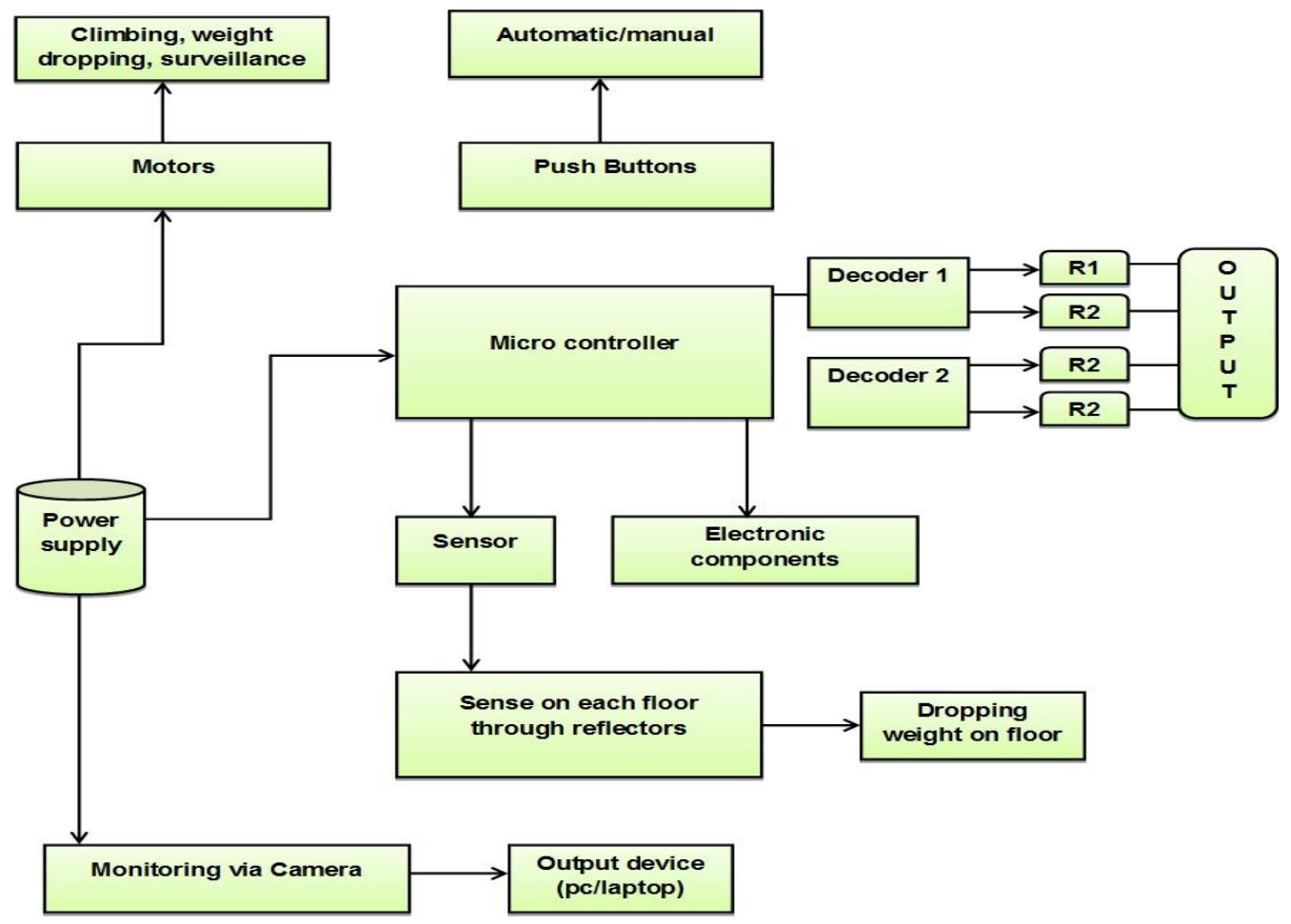

Fig. 2: System diagram of the proposed robot surveillance system 


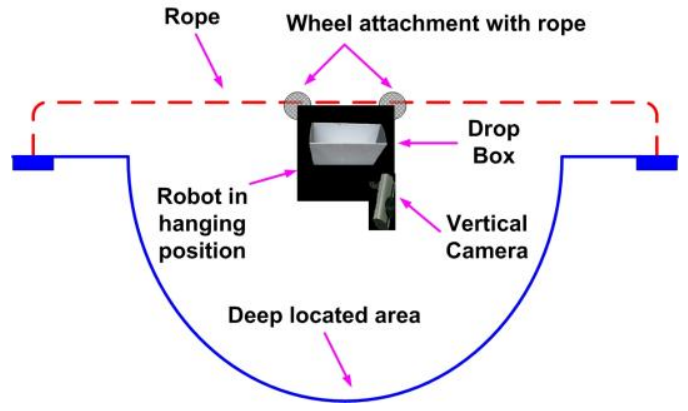

Fig. 3: Horizontal movement application of the robot in deep located areas

As indicated in the previous paragraphs, a camera is mounted on the top of the robot which gives life streaming of the floors it traverse. The video transmission is continuous whether it is operating in automatic or manual mode. Various images from the stream video transmitted by the robot are shown in Fig. 4. As it is evident from the pictures that stream videos are quite clear and stable. Jitter in the video is minimal and that is mainly due to the wireless channel between the transmitter and receiver. The overall flow diagram of the system and the state diagram are shown in Fig. 5. A detailed description of both hardware and software being used with their interconnection is presented in the next section.

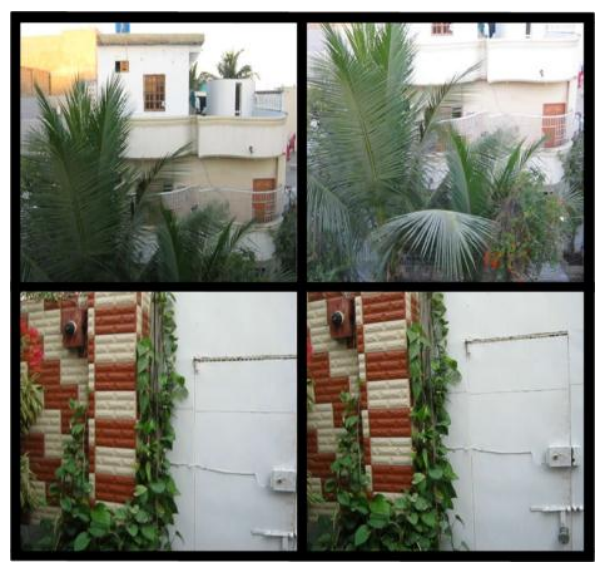

Fig. 4: Stable video streams transmitted by the RF transmitter from the robot to the PC terminal he designed robot under vertical and horizontal movements

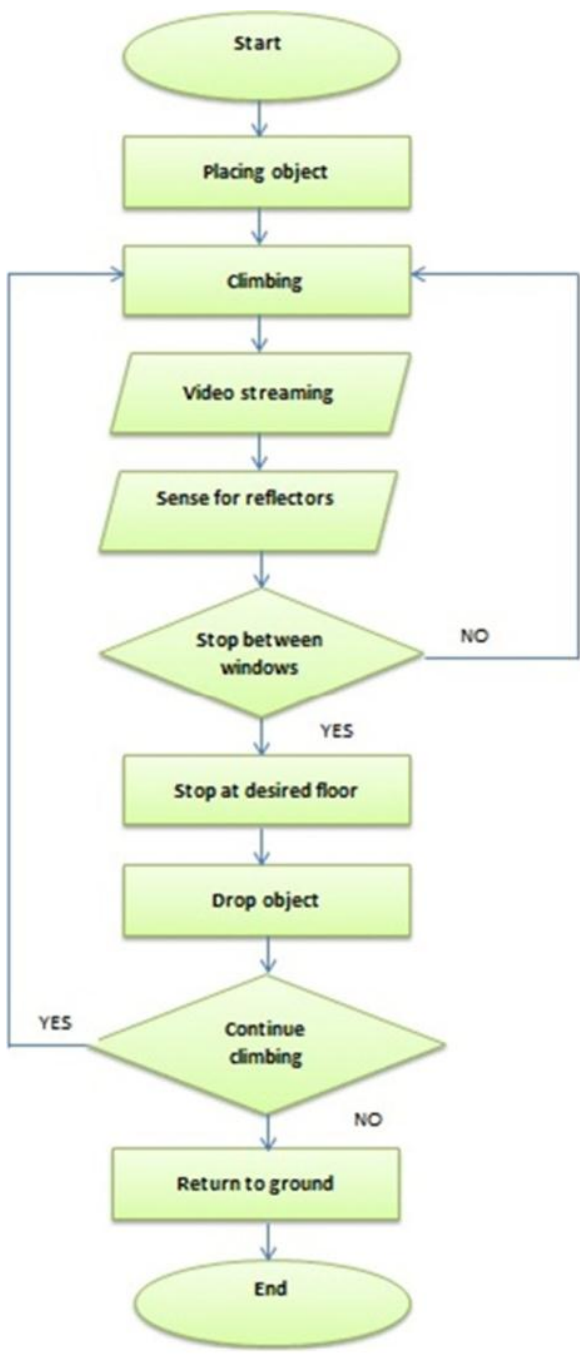

(a)

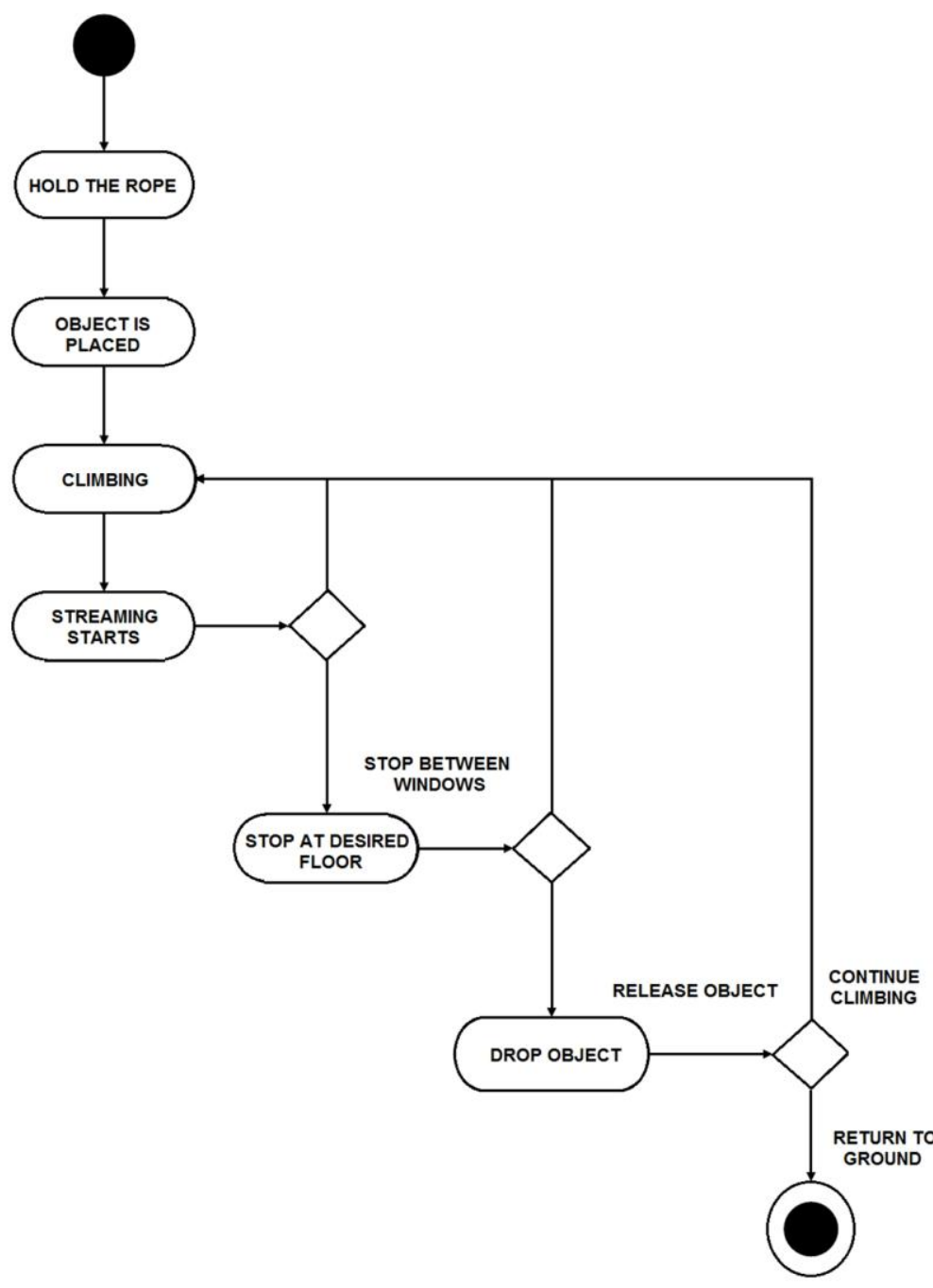

(b)

Fig. 5: (a) Data flow diagram of the proposed system (b) State diagram of the proposed system 


\section{Software and Hardware Component Description and Interconnection}

Basically most of the components being used are hardware components. They are either mechanical or electrical parts. Starting with the body of the robot itself, the robot is a rectangle in shape. At the front of the robot two major areas can be seen. The first one is the blue strip panel at the bottom containing some switches for manual and automatic mode of operation as shown in Fig 6 (a). These switches are connected to the actual circuitry of the robot and located behind a transparent area. In addition at the top, two wheels can be seen which are used for vertical climbing. A detailed description of these wheels will be given later. Now from the side view, the inner components can be seen as shown in Fig. 6 (b). It is clear that a third wheel can be seen in the middle of the robot which is used for vertical movement of the robot. Furthermore, note that the middle wheel is actually a composite of two disks. The two disks interconnected with a white cylindrical shaped material. Both disks are attached to the front and back board which are of Backlite material. Backlite material is light and has many features and advantageous especially in construting body of robots used for climbing purposes. In addition, looking at the gap between the front and back panels, the electrical circuitry is clear. At the bottom, we can see two DC batteries of 6 Volts each to provide power to the circuitry and motors attach to the wheels. Two sets of chains can also be seen which are attached to each wheel.

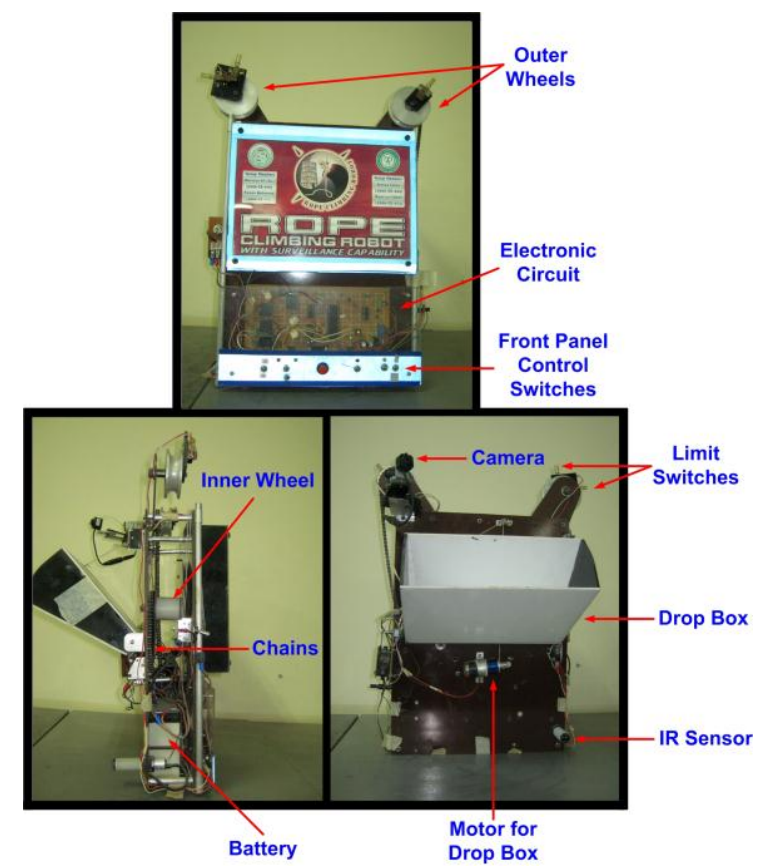

Fig. 6: Robot view from front, side and back with major components being used

Now going towards the back panel a wireless camera for surveillance can be seen as shown in Fig. 6 (c). Furthermore a huge drop box capable of holding objects till $1 \mathrm{Kg}$ weight can be seen which is controlled by a motor below it for sliding the drop box up and down. At the bottom right of the robot, IR sensor is used for sensing a strip. Note that at each floor, a sensing strip shown in Fig. 7 (a) is used so that the IR sensor is able to sense it and according drop the object. The methodology of IR sensor which is used here is the Luster recognition in which detection is not affected by target color and transparent targets can be detected. In this mode when the light beam hits a target, the beam reflects differently according to the luster of the target. The sensor detects the difference in luster based on how the beam reflects light as shown in Fig. 7 (b). It is worth mentioning here that sensing is done through an IR sensor just to detect that a specific floor is reached when automatic mode of operation is used. Although such floor detection can be done by the camera itself through computer vision and image processing techniques but since our objective was only to make a robot capable of climbing ropes, other aspects such as processing surveillance images for automatic detection of floors was not our targeted objective. Figures 8 and 9 shows a close up look of some of the above mentioned components including the switching panel, wireless camera, the three wheels ( 2 for horizontal and one for vertical movement), the IR sensor, the motor responsible for controlling the drop box and the drop box itself.

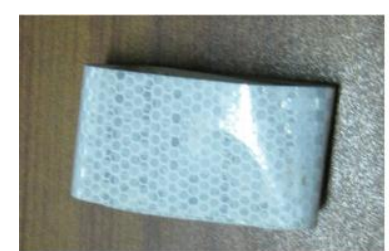

(a)

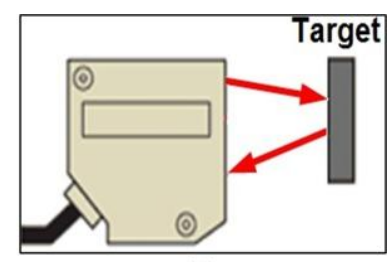

(b)
Fig. 7: (a) The strip sensor used at each floor detected by the IR sensor (b) The differentiated luster mode of operation used by the IR sensor

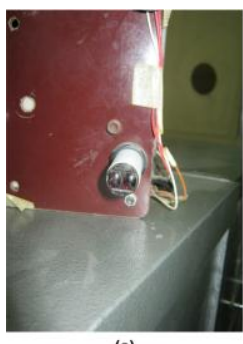

(a)

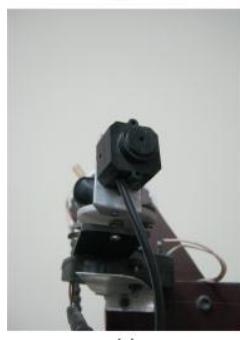

(c)

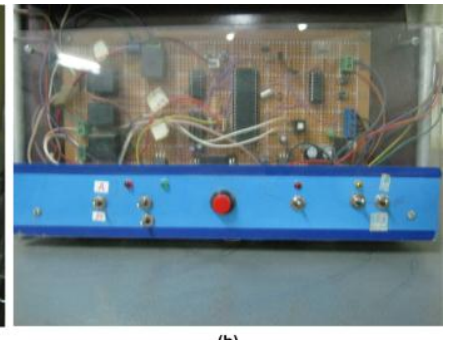

(b)

(d)

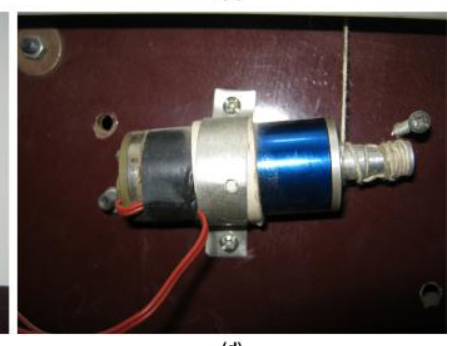

Fig. 8: Various components of the robot which includes (a) IR sensor (b) Front switching control panel (c) Wireless camera (d) Motor for drop box opening and closing mechanism 


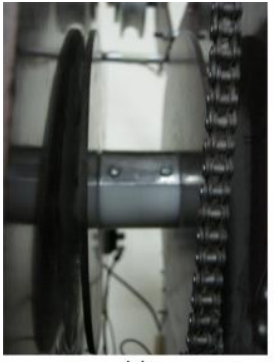

(a)

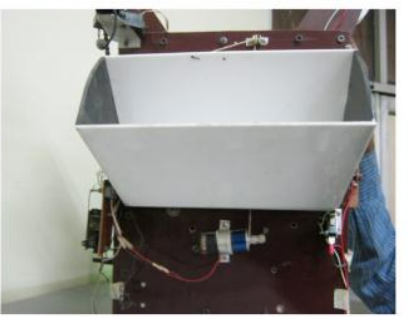

(c)

Fig. 9: Various components of the robot which includes (a) Middle wheel from backlite material to control the vertical movement of the robot (b) Upper wheels to control the horizontal movement of the robot (c) The drop box

Another feature we used in the design of the robot is the use of limit sensors at the top, bottom and across. The limit sensors as shown in Fig. 10 are used to sense the end of the floor. When reaching the top floor and the limit sensor touches a platform that we need to have at the end of a floor. Upon touching the floor a reverse mechanism of the motor is going to be activated and the robot will move in the reverse direction. Similarly when the robot reaches the bottom and the limit sensors at the bottom touch the earth or a platform the movement mechanism stops. The reverse direction movement and movement stop at the bottom after colliding with the limit sensors is shown in Fig. 10 (b-c). In a similar manner horizontal limit sensors work in the same manner.

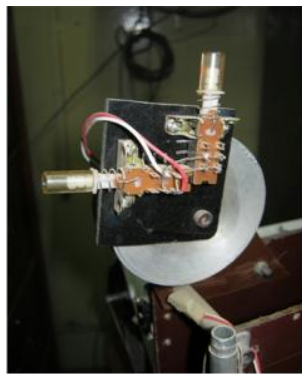

(a)

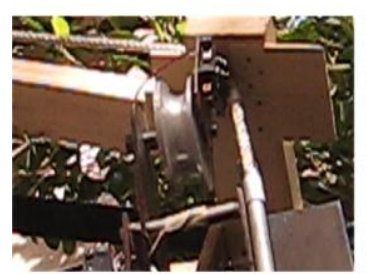

(b)

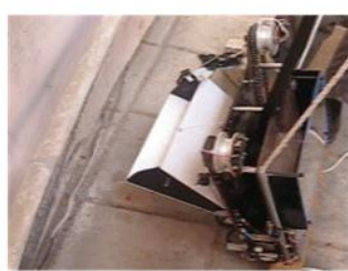

(c)
Fig. 10: (a) Limit switches for horizontal and vertical movement termination (b) A snapshot of the robot stopping by the vertical upper limit switches (c) A snapshot of the robot stopping by the vertical lower limit switches
Now to discuss the movement of wheels it is important to clarify that there are two types of ropes being used. As shown in Fig. 11 and indicated in the above paragraphs that whether we are talking about vertical or horizontal movements the device which is responsible for the rotation of the rope over the wheels is a motor. This motor is called the Porsche motor. The Porsche motor as shown in Fig. 11 (a) is above the two batteries. With this motor there are two chains attached to it, one for the horizontal movement and the other for the vertical movement. When the robot is moving in the vertical direction as shown in Fig. 11 (b), the two parallel ropes at the far ends are simply used for support and are attached behind the two wheels on top. The main rope which is black in color and going through the middle of the robot is responsible for pulling the robot upwards or bringing it downwards. The front view of the rope being used as shown in Fig. 11 (c) has a hook type which in turn is attached to another hook mounted on the middle wheel shown in Fig. 11 (d). The attachment point is shown in Fig. 11 (e). The rope is pulled upwards and being rested on a metal rod on the top of the robot for a support as shown in Fig. 11 (f). On the other hand for the horizontal movement, there is no need for the black rope. Only one rope is used and the robot is in hanging position on top of the two wheels as shown in Fig. 11 (g,h).

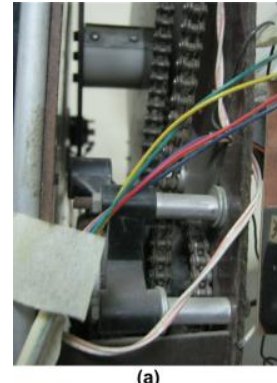

(a)

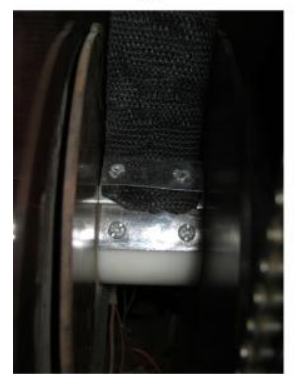

(e)

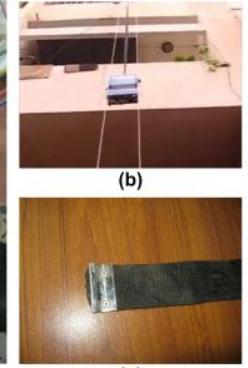

(c)

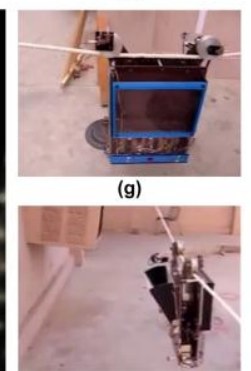

(h)
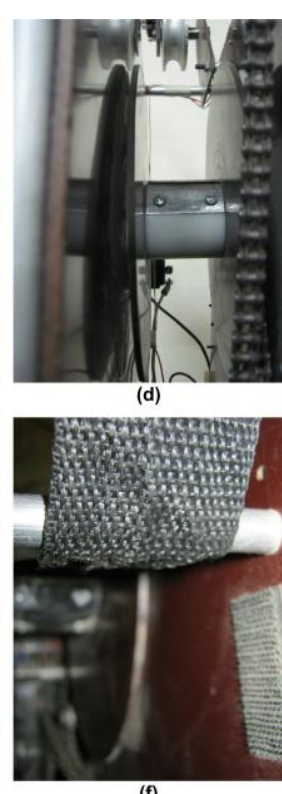

Fig. 11: Various components and functions of the robot which includes (a) Two chains attached to the Porsche motor for horizontal and vertical wheels control (b) The two ropes and the middle black rope being used for vertical movement (c) The black rope used for vertical movement with a buckle latch in from (d) A second buckle on the middle wheel (e) The connection of both buckles (f) The black rope when pulled up resting on a metal rod at the top of the robot (g-f)

Two snapshots of the robot in hanging position while moving horizontally

Now referring to the actual electrical circuitry a microcontroller is used to control and synchronize all the operations and electronic components being used such as relays, switches, RF module, motors and 
sensors. In our robot, we have used the Microchip PIC16F87X which is a powerful yet easy-to-program [15]. The microchip features 256 bytes of EEPROM data memory, self-programming, an ICD, 2 Comparators, 8 channels of 10-bit Analog-to-Digital (A/D) converter, two capture/compare/PWM functions, the synchronous serial port can be configured as either 3-wire Serial Peripheral Interface (SPI ${ }^{\mathrm{TM}}$ ) or the 2-wire Inter-Integrated Circuit $\left(\mathrm{I}^{2} \mathrm{C}^{\mathrm{TM}}\right)$ bus and a Universal Asynchronous Receiver Transmitter (USART). All of these features make it ideal for more advanced level A/D applications in automotive, industrial, appliances and consumer applications.

In addition 4 relays are used to control the vertical, horizontal movements and the drop box mechanism. Relays allow one circuit to switch a second circuit which can be completely separate from each other. In addition an RF module is used for receiving signal from an RF transmitter in case of manual control. We have used a $433 \mathrm{MHz}$ RF transmitter module. It has a sensitivity of $3 \mathrm{uV}$. The RWS-434 receiver operates from 4.5 to 5.5 volts-DC and has both linear and digital outputs.

Finally, in term of software, we have only used visual basic for programming of the manual and automatic mode of operation and for the drop mechanism. The code we have implemented is given in the Appendix.

\section{Performance Analysis of the Designed Robot}

The robot was tested at various places and heights. The robot was tested up till $7^{\text {th }}$ floor vertically and horizontally till $300 \mathrm{ft}$. Video transmission was quite stable throughout the movement. In addition the movement itself was smooth and at constant speed. Recognition of strips by the sensor was perfect and the robot paused for about fifteen seconds at each floor to permit enough time for the drop mechanism to take place. Of course the pause time can be modified in the code. So in terms of movement and other features discussed earlier, the robot was showing a very good performance. We tested the robot in 10 different places.

Table 1: Cost of Components of the Robot

\begin{tabular}{|l|c|}
\hline Components & Cost in USD \\
\hline Micro Controller & 2 \\
\hline DC Motor dropping & 10 \\
\hline DC Motor (Porsche) & 120 \\
\hline IR Sensors & 4 \\
\hline RF Modules & 15 \\
\hline Decoders & 2 \\
\hline Relays & 6 \\
\hline Electronic Components & 10 \\
\hline Remote Control & 20 \\
\hline Mechanical Charges & 30 \\
\hline Battery & 10 \\
\hline Camera & 35 \\
\hline Miscellaneous & 20 \\
\hline Total & $\mathbf{2 8 4}$ \\
\hline
\end{tabular}

In terms of cost, Table 1 shows a rough estimation of the robot. The average cost of this robot is around below 300 USD which is a reasonable amount compared to other rope climbing robots.

\section{Conclusion}

In our work we designed and developed a rope climbing robot capable of moving vertically and horizontally. Mainly it is used to drop objects at different locations such as floors, construction sites, deep holes, etc. Furthermore, the robot has a mounted camera which can be used for survillenc purpose. The video transmitted by the robot in movement to the computer is stable. In addition the sensing mechanism is quite perfect as in order to drop an object, the robot has to pause for some time for the dropping to take place. This is done through sensing strips at each floor. Furthermore, the design of the robot is efficient in terms of the components being used and the overall performance of the robot. It is also very cost effective as compare to various rope climbing robots. Finally as future work, significant amount of intelligence can be incorporated in our robot such as to pause at floors not by using sensors but using vision. The camera can send an image of the floor to the server and based on computer vision and image processing techniques, the scene can be analyzed and recognized. In addition, we can combine both horizontal and vertical movement at 
the same time in both manual and automatic mode of operation. There might be more than one apartment in a floor. So the initial and main movement could be vertical while the secondary movement could be horizontal.

\section{Appendix \\ (CODE FOR ROBOT MANUAL AND AUTOMATIC TRANSMISSION)}

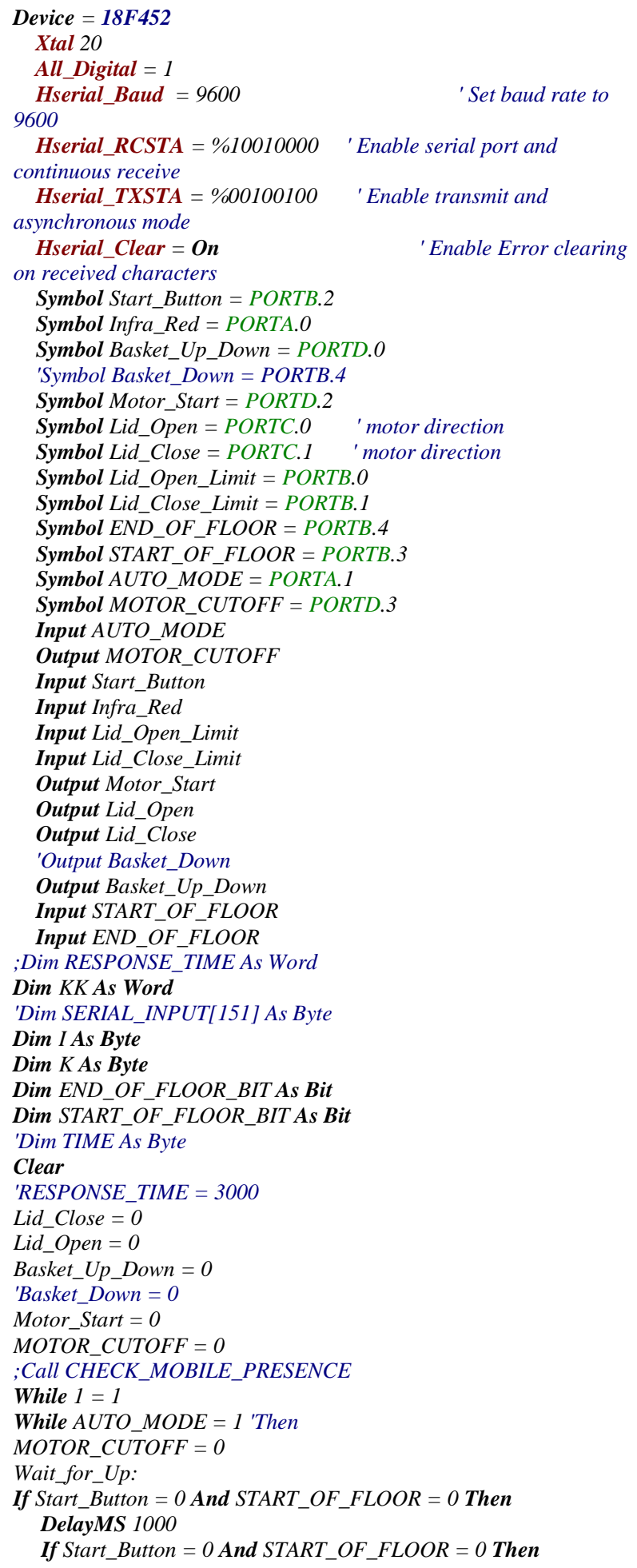

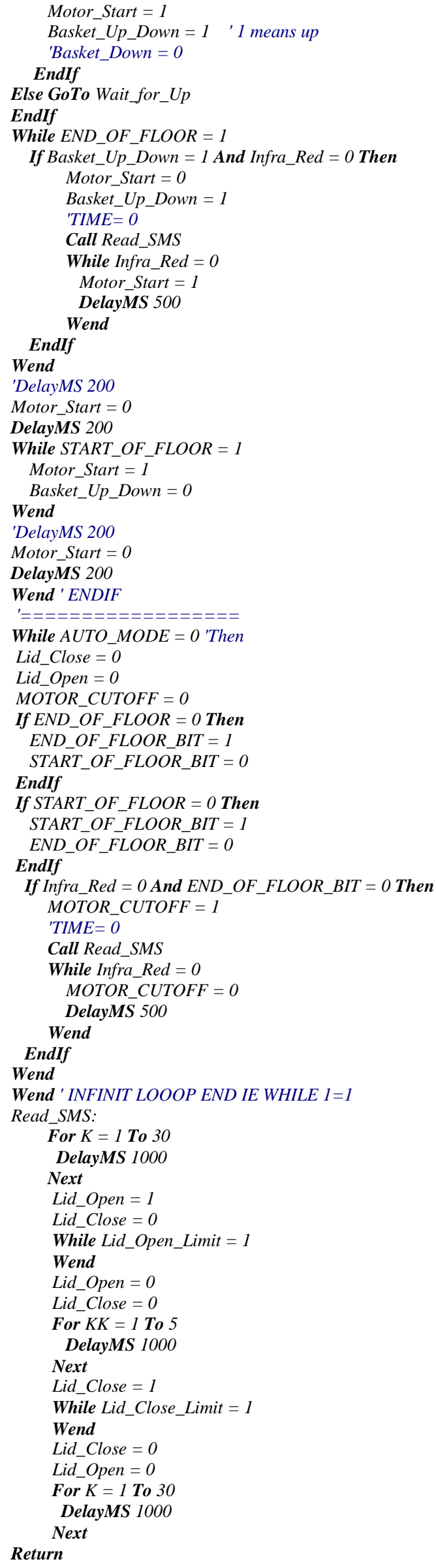

I.J. Intelligent Systems and Applications, 2013, 09, 1-9 


\section{References}

[1] Kemp C. C., Edsinger A. and Torres-Jara E., Challenges for Robot Manipulation in Human Environments, IEEE Robotics \& Automation Magazine, vol. 14, no. 1, pp. 20 - 29, Mach 2007.

[2] Brian Day, Cindy Bethel, Robin Murphy and Jennifer Burke, A Depth Sensing Display for Bomb Disposal Robots, in proc. of the IEEE International Workshop on Safety, Security and Rescue Robotics 2008, Sendai, Japan, pp. 146 151, October 2008.

[3] Bi-qiang Yao and Guang Wen, "Research on the tele-operation robot system with tele-presence, in proc. of the IEEE International Conference on Mechatronics and Automation (ICMA 2011), Beijing, China, pp. 2308 - 2311, August, 2011.

[4] Juhyun Park, Jeonghun Choi, Myoungheum Park, Sukwon Hong and Hyomin Kim, A Study on Intelligent Video Security Surveillance System with Active Tracking Technology in Multiple Objects Environment, International Journal of Security and Its Applications, vol. 6, no. 2, pp. 211 -216, April 2012.

[5] Alison Wakefield, The Public Surveillance Functions of Private Security, Surveillance \& Society, vol. 2, no. 4, pp. 529-545, 2004.

[6] Izquierdo E., Knowledge-Based Image Processing for Classification and Recognition in Surveillance Applications, in proc. of the IEEE International Conference on Image Processing 2006, Atlanta, USA, pp. 2377 - 2380, October 2006.

[7] Hu W., Tan T., Wang L. and Maybank S., A Survey on Visual Surveillance of Object Motion and Behaviors, IEEE Transactions on Systems, Man, and Cybernetics, Part C: Application and Review, vol. 34, no. 3, pp. 334 - 352, August 2004.

[8] Robert Bodor, Andrew Drenner, Paul Schrater and Nikolaos Papanikolopoulos, Optimal Camera Placement for Automated Surveillance Tasks, Journal of Intelligent and Robotic Systems, vol. 50, no. 5, pp. 257 - 295, November 2007.

[9] Mark Micire, Evolution and field performance of a rescue robot, Journal of Field Robotics, vol. 25, no. 1-2, pp. 17 - 30, January 2008.

[10] Xiang Li, Sridharan M. and Shiqi Zhang, To look or not to look: A hierarchical representation for visual planning on mobile robots, in proc. of the IEEE International Conference on Robotics and Automation (ICRA) 2011, Shanghai, China, pp. 6239 - 6244, May 2011.

[11] Soofiyani F.R., Rahmani A.M. and Mohsenzadeh M., A Straight Moving Path Planner for Mobile Robots in Static Environments Using Cellular Automata, in proc. of the 2nd International
Conference on Computational Intelligence, Communication Systems and Networks, Liverpool, United Kingdom, pp. 67 - 71, July 2010.

[12] Nadeesha Ranasinghe, Jacob Everist and Wei-Min Shen, Modular Robot Climbers, in proc. of the IEEE/RSJ International conference on Intelligent Robots and Systems, San Diego, USA, November 2007.

[13] R Liu, G Zong, H Zhang and X Li, A Cleaning Robot for Construction Out-wall with Complicated Curve, in proc. of the Sixth International Conference on Climbing and Walking Robots and their Supporting Technologies for Mobile Machines (CLAWAR 2003), Catania, Italy, pp. 825 - 834, September 2003.

[14] Matteo Zoppi and Rezia M. Molfino, Multi-legged multi-roped walking and climbing robots: online static equilibrium analysis, Advanced Robotics, vol. 20, no. 2, pp. $165-180,2006$.

[15] Datasheet:

(http://ww1.microchip.com/downloads/en/deviced oc/30292c.pdf)

\section{Authors' Profiles}

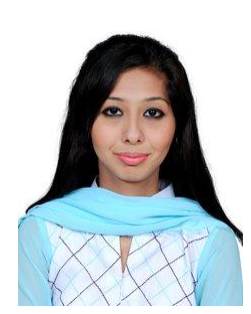

Kanza Zafar received her BS in Computer Engineering from Sir Syed University of Engineering and Technology (SSUET), Karachi, Pakistan. At present she is working as Research Assistant in the Department of Computer Engineering. Her research interest includes Robotics, communication systems and wireless networks.

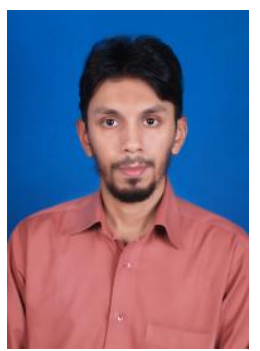

Ibrahim M. Hussain obtained his MS in Computer Engineering with specialization in Computer Networks from the Sir Syed University of Engineering and Technology (SSUET), Karachi, Pakistan and B.E. in Computer Engineering from N.E.D. University of Engineering and Technology. At present, he is working as an Assistant Professor in the Department of Computer Engineering, SSUET. His research interest includes wireless communication and networks, wireless networks design and signal and image processing. He has number of research publication in international journals and conferences. 